'Departamento Gastroenterología y Hepatología, Escuela de Medicina Pontificia Universidad Católica de Chile. Santiago, Chile.

2Departamento Gastroenterología y Hepatología, Clínica Universidad de los Andes. Santiago, Chile.

${ }^{3}$ Sección Diabetes, Hospital Clínico Universidad de Chile. Santiago, Chile. ${ }^{4}$ Centro de Diabetes Adulto, Clínica Las Condes. Santiago, Chile. ${ }^{5}$ Departamento Diabetes Hospital Padre Hurtado, Facultad Medicina Clínica Alemana-Universidad del Desarrollo. Santiago, Chile. ${ }^{6}$ Unidad de Diabetes y Nutrición, Clínica Universidad de los Andes. Santiago, Chile. ${ }^{7}$ Departamento Diabetes, Hospital San Juan de Dios, Universidad de Chile y Clínica Las Condes. Santiago, Chile. ${ }^{8}$ Departamento de Gastroenterología, Clínica Santa María. Santiago, Chile. ${ }^{9} \mathrm{Centro}$ integral de enfermedades del hígado, HepatoMed. Santiago, Chile.

${ }^{10}$ Departamento Endocrinología y Nutrición, Clínica Sanatorio Alemán. Concepción, Chile.

11Departamento de Nutrición, Diabetes y Metabolismo, Facultad de Medicina. Pontificia Universidad Católica de Chile. Santiago, Chile.

${ }^{12}$ Departamento Gastroenterología Hospital Clínico Universidad de Chile,

Clínica Dávila. Santiago, Chile.

${ }^{13}$ Departamento GastroenterologíaHepatología Hospital Clínico Universidad de Chile. Santiago, Chile. ${ }^{14}$ Depto. Medicina Interna, Facultad de Medicina, Universidad de Concepción. Concepción, Chile.

${ }^{15}$ Departamento Diabetes, Universidad de Antofagasta. Santiago, Chile.

Recibido el 9 de septiembre de 2020, aceptado el 26 de octubre de 2021.

Correspondencia a: Dr. Juan Pablo Arab

Departamento de Gastroenterología Escuela de Medicina, Pontificia Universidad Católica de Chile. Marcoleta \#367 Santiago, RM, Chile. jparab@uc.cl

\section{Resumen ejecutivo: Enfermedad por hígado graso no alcohólico en sujetos con diabetes mellitus tipo 2: Postura conjunta de la Asociación Chilena de Hepatología (ACHHEP) y la Sociedad Chilena de Diabetología (SOCHIDIAB)}

\author{
JUAN PABLO ARAB ${ }^{1}$, LORENA CASTRO ${ }^{2}$, \\ PATRICIA C. GÓMEZ ${ }^{3,4}$, PAULINA VIGNOLO ${ }^{5}$, \\ MARCO ARRESE ${ }^{1}$, FRANCISCO BARRERA ${ }^{1}$, \\ FABIOLA CASTRO ${ }^{2}$, LUIS ANTONIO DÍAZ ${ }^{1}$, ANIBAL DONOSO ${ }^{6}$, \\ KARINA ELGUETA ${ }^{7,4}$, KATHERINE GONZÁLEZ ${ }^{8,9}$, \\ MARÍA ISABEL GONZÁLEZ ${ }^{10}$, MANUEL MORENO ${ }^{11}$, \\ RAÚL LAZARTE ${ }^{3,8,12}$, JAIME PONIACHIK ${ }^{3,8}$, \\ PATRICIO SALMAN ${ }^{14}$, JUAN PATRICIO VALDERAS ${ }^{15}$

\section{Non-alcoholic fatty liver disease in type 2 diabetes: a joint statement of the Chilean Hepatology and Diabetology Societies}

Non-alcoholic fatty liver disease (NAFLD) has a high prevalence and risk of progression to cirrhosis and other complications in patients with type 2 diabetes mellitus (T2DM). Likewise, the presence of NAFLD implies a high risk of developing T2DM, determining a bidirectional relationship between them. The diabetology and hepatology societies, developed a joint initiative aiming to unify criteria, reviewing the definitions, diagnostic criteria, risk stratification, treatment, and follow-up of patients with NAFLD and T2DM. The key questions to be discussed were defined by a panel of specialists in diabetology and hepatology. The Delphi methodology was used to reach consensus on the respective recommendations. Based on the discussion generated among the experts, diagnostic and treatment algorithms were proposed, as well as an indication for referral and the role of the different specialists involved in the management of these patients. Strengthening multidisciplinary work with patients with NAFLD and T2DM will allow the early recognition of the disease, the prevention of the progression to cirrhosis, and reducing the associated complications.

(Rev Med Chile 2021; 149: 1360-1371)

Key words: Diabetes Mellitus, Type 2; Fatty Liver; Liver Cirrhosis; Non-alcoholic Fatty Liver Disease. 
L a enfermedad por Hígado Graso No Alcohólico (HGNA) tiene una alta prevalencia complicaciones en la población diabética. Esto motivó a que ambas sociedades, ACHHEP y SOCHIDIAB, tuvieran como objetivo fortalecer el conocimiento médico sobre el manejo integral de este binomio. Se trabajó con especialistas de ambas sociedades quienes respondieron en conjunto ocho preguntas, sugirieron algoritmos de diagnóstico y manejo, emitiendo recomendaciones que fueron votadas y acordadas por método Delphi. Se describe a continuación un resumen ejecutivo de estas, orientado a médicos y profesionales de la salud que evalúan y tratan a estos pacientes.

\section{1. ¿Cómo se define en la actualidad el HGNA y cuál es su relevancia en la población diabética?}

La enfermedad hepática por hígado graso no alcohólico (HGNA) se define por la presencia de esteatosis en más de 5\% de los hepatocitos, en ausencia de consumo de alcohol u otras causas secundarias. Su prevalencia se estima en 25\% de los adultos ${ }^{1}$. Tradicionalmente el HGNA se ha subdividido en esteatosis aislada cuando sólo hay esteatosis, y en estetohepatitis no alcohólica (EHNA) cuando presenta esteatosis, inflamación y balonización hepatocelular ${ }^{2}$. La presencia de fibrosis hepática es el principal factor pronóstico de desarrollo de cirrosis y mortalidad de causa hepática en HGNA3 ${ }^{3}$.

Se encuentra en debate el cambio del nombre de HGNA a Metabolic-(dysfunction) Associated Fatty Liver Disease (MAFLD) $)^{4,5}$.

En diabetes tipo 2 (DM2), la prevalencia de HGNA (50-70\%), cirrosis (2-6\%) y riesgo de hepatocarcinoma (HCC) es significativamente mayor que en la población general ${ }^{6}$. El HGNA es una de las complicaciones más frecuentes en DM2, superando la prevalencia de nefropatía, retinopatía y neuropatía diabética. A su vez el HGNA duplica la probabilidad de desarrollar DM2 ${ }^{6}$. La presencia de HGNA y DM2 son potentes factores de riesgo de eventos cardiovasculares (FRCV), siendo ésta la principal causa de muerte en ambas patologías ${ }^{1,7}$.

\section{Recomendaciones}

1. Los sujetos con DM2 presentan una alta prevalencia de HGNA, EHNA, cirrosis y sus complicaciones. Se sugiere pesquisa activa de HGNA en esta población.

2. La presencia de HGNA supone un alto riesgo de desarrollo de DM2, determinando una relación bidireccional entre ambas patologías. Por lo que la DM2 debe ser diagnosticada y tratada en forma oportuna en sujetos con HGNA.

3. Los sujetos con coexistencia de DM2 e HGNA presentan un elevado riesgo de enfermedades cardiovasculares por lo que su evaluación y tratamiento a este respecto es fundamental.

\section{2. ¿Cómo se diagnostica el HGNA en diabéticos en la práctica clínica?}

Clínicamente el HGNA suele cursar en forma asintomática y las pruebas hepáticas pueden ser normales o presentar leve elevación de estas ${ }^{8}$.

Existen distintas formas de realizar la pesquisa, por medio de métodos de imagen como la ecografía abdominal, ampliamente disponible y de bajo costo, aunque con sensibilidad y especificidad variable, especialmente en población con obesidad ${ }^{6}$. Otras imágenes como tomografía computada (TC) o resonancia magnética (RM) son de gran aporte, pero no en la primera valoración. La utilización de la determinación de CAP (Controlled attenuation parameters) realizado por ultrasonido incorporado en la elastografía de transición es otra forma más recientemente implementada. Otro método de pesquisa, es a través de sistemas de puntuación serológicos de esteatosis. El liver fat score (NAFLD-LFS) y el Hepatic Steatosis Index (HSI) han demostrado buen rendimiento en pesquisa en población general ${ }^{9}$.

En caso de existir alteración de las enzimas hepáticas se debe completar el estudio etiológico y descartar otras causas de enfermedad hepática crónica así como asegurarse de una ingesta menor a $20 \mathrm{~g}$ de alcohol diario.

No existe consenso en las guías internacionales en realizar pesquisa de HGNA a todos los pacientes con DM2, pero pareciera ser recomendable su realización ${ }^{10}$. 


\section{Recomendaciones}

4. Se sugiere la pesquisa activa de HGNA es población diabética utilizando como herramienta la ecotomografía abdominal.

5. En sujetos diabéticos con elevación persistente de enzimas hepáticas con o sin esteatosis en la ecografía está indicado estudiar otras etiologías distintas al HGNA, valorar la posibilidad de coexistencia de enfermedades y en caso de duda realizar una biopsia hepática.

\section{3. ¿Qué importancia tiene la coexistencia de HGNA y diabetes?}

Pacientes con HGNA presentan EHNA en 30 a $40 \%{ }^{11}$. El principal factor de riesgo de HGNA es la DM2 ${ }^{12}$. La obesidad es predictor de altas tasas de progresión a fibrosis y la baja de peso en esta población se asocia a regresión de las alteraciones histológicas ${ }^{13}$. Otros factores de progresión son dieta alta en grasas saturadas y fructosa, SMet, resistencia a la insulina, edad mayor a 45-50 años, etnia hispánica y polimorfismos genéticos, en los genes PNPLA3 y TM6SF2 $2^{14,15}$. Un estudio, mostró asociación de FA (fibrosis avanzada) con DM2, hipertensión arterial, hipercolesterolemia e hipertrigliceridemia y asociación de cirrosis con DM2 e hipertrigliceridemia, ${ }^{16}$ sugiriendo un rol de la regulación lipídica en la progresión a fibrosis. Existen estudios de asociación entre gravedad de la fibrosis y cambios en la microbiota intestinal ${ }^{17}$. Es además conocida la relación sinérgica entre HGNA y consumo de alcohol en la progresión de la enfermedad hepática ${ }^{18}$, en contraposición, la actividad física tiene una relación inversa con la progresión de la fibrosis ${ }^{19}$.

\section{Recomendación}

6. Los pacientes con HGNA con comorbilidades tales como DM2, obesidad, SMet, resistencia a la insulina y/o dislipidemia tienen más riesgo de progresión a fibrosis, cirrosis y desarrollo de HCC, por lo que se debe identificar, manejar en forma adecuada la condición y monitorizar la fibrosis en forma estrecha en esta población.

\section{4. ¿Por qué y cómo evaluamos la fibrosis en la población diabética con HGNA?}

La estimación del grado de fibrosis hepática es clave para establecer el pronóstico del HGNA, dado que permite predecir la progresión a cirrosis, mortalidad de causa hepática y global, e incluso el riesgo de hepatocarcinoma (HCC $)^{20,21}$. La fibrosis hepática está presente en hasta 21\% de DM2, siendo la fibrosis avanzada en alrededor de 10\% de ellos. El gold standard es la biopsia hepática, método con limitaciones, en particular por su naturaleza invasiva con potenciales complicaciones, error de muestreo y costo. Múltiples pruebas no invasivas se han desarrollado para la evaluación de la fibrosis hepática. La mayoría se basa en parámetros clínicos y de laboratorio, o en imágenes considerando las propiedades físicas del hígado (Tabla 1). Los puntajes de NAFLD Fibrosis Score (NFS) y FIB-4 permiten descartar la presencia de fibrosis avanzada (FA) por su alto valor predictivo negativo ${ }^{2}$. Cabe destacar que el rendimiento de estos marcadores en DM2 es menor que en la población general, por lo que se debe mantener un alto índice de sospecha y utilizar un método basado en elastografía ${ }^{22,23}$ y en casos especiales, la biopsia hepática ${ }^{24}$.

\section{Recomendaciones}

7. NAFLD Fibrosis Score (NFS) y FIB-4 son sistemas de puntuación no invasivos útiles para la evaluación inicial de fibrosis en pacientes con HGNA y DM2, aunque su rendimiento es limitado y debe ser complementado con técnicas basadas en elastografía hepática en caso de dudas o sospecha clínica.

8. Cuando NFS y FIB-4 no permiten excluir fibrosis avanzada (cuando caen en la categoría de riesgo intermedio o alto) se debe utilizar un método basado en elastografía transitoria hepática controlada por vibración. Otras técnicas de elastografía tienen que ser utilizadas según disponibilidad.

9. Cuando los métodos de medición de fibrosis no invasivos, serológicos o imagenológicos, no permiten excluir la fibrosis avanzada o se sospecha que el paciente está progresando en el grado de fibrosis se debe considerar realizar una biopsia hepática. 
Tabla 1. Sistemas de puntuación no invasivos basados en marcadores bioquímicos y elastografía en EHGNA [Adaptado de (2)]

\begin{tabular}{|c|c|c|c|}
\hline $\begin{array}{l}\text { Sistema de } \\
\text { puntuación }\end{array}$ & Componentes & $\begin{array}{l}\text { Puntaje de corte } \\
\text { inferior (para excluir } \\
\text { fibrosis avanzada) }\end{array}$ & $\begin{array}{l}\text { Puntaje de corte } \\
\text { superior (para incluir } \\
\text { fibrosis avanzada) }\end{array}$ \\
\hline $\begin{array}{l}\text { NAFLD fibrosis score } \\
\text { (NFS) }\end{array}$ & $\begin{array}{l}\text { Edad, glicemia de ayunas alterada/ } \\
\text { diabetes, IMC, plaquetas, albúmina, } \\
\text { relación AST / ALT }\end{array}$ & $\begin{array}{l}<-1,455 \text { (36-64 años) } \\
<0,12 \text { (65 años o más) }\end{array}$ & $>0,675$ \\
\hline FIB-4 & Edad, AST, ALT, Plaquetas & $\begin{array}{l}<1,3 \text { (36-64 años) } \\
<2 \text { (65 años o más) }\end{array}$ & $>2,67$ \\
\hline $\begin{array}{l}\text { Fibroscan } \\
\text { - Sonda M } \\
\text { - Sonda XL }\end{array}$ & $\begin{array}{l}\text { Elastografía transitoria controlada } \\
\text { por vibración }\end{array}$ & $\begin{array}{l}<5,8 \\
<4,8\end{array}$ & $\begin{array}{l}>8,7 \mathrm{kPa} \\
>5,7 \mathrm{kPa}\end{array}$ \\
\hline $\begin{array}{l}\text { Shear-wave elastography } \\
\text { (SWE) }\end{array}$ & $\begin{array}{l}\text { Elastografía de ondas de corte en } \\
\text { 2D y Acoustic Radiation Force } \\
\text { Impulse (ARFI) }\end{array}$ & $<2,6$ & $>3,02 \mathrm{~m} / \mathrm{s}$ \\
\hline $\begin{array}{l}\text { Magnetic resonance } \\
\text { elastography (MRE) }\end{array}$ & $\begin{array}{l}\text { Elastografía por resonancia } \\
\text { magnética (ERM) }\end{array}$ & $<3,4$ & $>3,62 \mathrm{kPa}$ \\
\hline
\end{tabular}

*Calculadoras en línea: NFS: https://nafldscore.com. FIB-4 y APRI: https://www.rccc.eu/calculadoras/Fib4.htm.

\section{5. ¿Cuál es el rol de la dieta, ejercicio y cirugía} bariátrica en el manejo del HGNA en diabéticos?

El manejo nutricional se enfoca en reducir el peso corporal y mejorar la sensibilidad insulínica. Una pérdida ponderal por sobre $5 \%$ disminuye la esteatosis y mejora la sensibilidad insulínica. Reducciones sobre 7-10\% mejoran la fibrosis ${ }^{25}$. La dieta debe ser individualizada, hipocalórica, considerando comorbilidades y adherencia a largo plazo. Se recomienda reducción calórica entre 500 a $1.000 \mathrm{kcal} /$ día, con aporte de 50-60\% de carbohidratos, $20-25 \%$ de grasas (saturadas $<10 \%$ ), $20-30 \%$ proteínas y alta en fibra. La dieta mediterránea, alta en grasas monoinsaturadas, omega 3 y polifenoles, y baja en alimentos procesados y fructosa, ha demostrado mejoría de la esteatosis hepática ${ }^{26}$. Se recomienda evitar colaciones, ya que podrían aumentar el contenido de grasa hepática e intraabdominal ${ }^{3}$. Los cambios de estilo de vida pueden lograrse con el apoyo de psicoterapia y manejo conductual. Se debe promover la abstinencia de alcohol en pacientes con HGNA, en particular con EHNA y cirrosis, por su asociación con progresión de la enfermedad y el desarrollo de $\mathrm{HCC}^{27}$. El café ha demostrado una relación inversa con la presencia de fibrosis en pacientes con $\mathrm{HGNA}^{28}$.
El ejercicio físico, tanto el aeróbico y de resistencia, reducen la esteatosis independiente de la reducción ponderal. La recomendación es realizar al menos 150-300 minutos semanales de intensidad moderada, distribuido en al menos 3 días $^{29}$.

La cirugía bariátrica es el método más efectivo en reducir de manera significativa y mantenida el peso, permitiendo una mejoría de las complicaciones y de la mortalidad de los pacientes con DM2 y obesidad que cumplan los criterios estándares ${ }^{30}$. Las técnicas más empleadas son la gastrectomía vertical en manga y el bypass gástrico en $\mathrm{Y}$ de Roux. Su realización requiere una adecuada selección de pacientes dentro de un equipo multidisciplinario que involucre un cambio de hábitos alimentarios y de ejercicios que permitan evitar la reganancia de peso y la aparición de comorbilidades asociadas.

\section{Recomendaciones}

10. La reducción de peso corporal mantenida, sobre 7-10\% y a sea con dieta hipocalórica y mediterránea, ejercicio, manejo conductual y en algunos casos cirugía bariátrica representan estrategias eficaces para el manejo de pacientes con obesidad, DM2 e HGNA con algún grado de fibrosis. 
11. En pacientes con HGNA, en particular los con EHNA y cirrosis, se debe promover la abstinencia de alcohol. En aquellos con esteatosis hepática aislada, el consumo de alcohol debe evitarse o estar estrictamente limitado a $30 \mathrm{~g} /$ dia en hombres $y<20 \mathrm{~g} /$ día en mujeres.

12. Se recomienda el manejo de estos pacientes por un equipo multidisciplinario (médico, nutricionista, psicólogo, kinesiólogo, etc.).

\section{6. ¿Cúal es el rol de las drogas antidiabéticas en} la evolución del HGNA en diabéticos? (Tabla 2)

\section{Insulino-sensibilizadores}

Metformina se asoció con reducción significativa en el índice de masa corporal (IMC) comparada con placebo, los niveles de aminotransferasas, el colesterol y la glucosa en ayunas. Sin embargo, los parámetros histológicos no se modifican significativamente ${ }^{31}$. Estudios recientes sugieren que su uso en pacientes con HGNA y FA se asocia con mayores tasas de supervivencia, menores tasas de descompensación y un riesgo reducido de $\mathrm{HCC}^{32,33}$.

Pioglitazona es una tiazolidinediona ampliamente estudiada en HGNA. Cinco ensayos controlados aleatorios muestran resultados consistentes en la resolución de la EHNA, de la balonización hepatocitaria o la inflamación, mientras que en algunos de los estudios se observó una mejoría de la fibrosis ${ }^{34-36}$. En cuanto a su seguridad, el aumento de peso (media, $4,4 \mathrm{~kg}$ ), la pérdida ósea en mujeres, y hallazgos controvertidos con respecto a su papel en el riesgo de cáncer de vejiga son elementos que limitan su uso ${ }^{36}$. No se encuentra disponible en nuestro país.
Análogos del receptor de GLP1 (GLP-1 AR)

Su uso en DM2 resulta en baja de peso, optimización de la presión arterial sistólica, mejoría en la $\mathrm{HbA} 1 \mathrm{c}$ e insulino-resistencia, con beneficio cardiovascular y renal ${ }^{37}$. Los estudios para evaluar su efecto en el HGNA sugieren efectos positivos. Un metaanálisis reciente de Mantovani y $\operatorname{cols}^{38}$, apoya la eficacia de estos (especialmente liraglutida y semaglutida) en la mejoría de HGNA. Estos estudios documentan que el uso de GLP1-RA mejora los niveles de enzimas hepáticas, favorece la regresión de la esteatosis hepática y de la balonización hepatocitaria, sin efecto robusto sobre la fibrosis hepática ${ }^{38-40}$. No hay datos de grandes estudios controlados para valorar su eficacia en el largo plazo. El costo y problemas en la tolerabilidad podrían ser una barrera para su uso.

\section{Inhibidores del co-transportador sodio glucosa 2 (iSGLT2)}

Tienen documentado beneficio cardiovascular y renal en pacientes con y sin diabetes. Revisiones sistemáticas recientes ${ }^{41}$ sugieren que su uso se asocia a efectos beneficiosos en HGNA, disminuyendo los niveles plasmáticos de ALT, GGT y esteatosis hepática. Sin embargo, no se dispone de evidencia robusta.

\section{Inhibidores de enzima dipeptidyl peptidasa 4 (iDPP4)}

Estudios evidencian que disminuyen significativamente la ALT, sin embargo, no se observaron cambios en los niveles de AST ni GGT. Tampoco se observaron beneficios en la fibrosis, esteatosis hepática ni en el IMC ${ }^{42}$.

Tabla 2. Resumen de los efectos de fármacos antidiabéticos en el HGNA

\begin{tabular}{|lccccc|}
\hline Fármacos & $\begin{array}{c}\text { Efectos en niveles de } \\
\text { enzimas hepáticas } \\
\text { (disminución) }\end{array}$ & $\begin{array}{c}\text { Cambios en la } \\
\text { histología } \\
\text { Esteatosis }\end{array}$ & $\begin{array}{c}\text { Cambios en la } \\
\text { histología } \\
\text { Inflamación }\end{array}$ & $\begin{array}{c}\text { Cambios en la } \\
\text { histología } \\
\text { Fibrosis }\end{array}$ & $\begin{array}{c}\text { Efectos en el } \\
\text { IMC }\end{array}$ \\
Metformina & Variable & Leve/nulo & Leve/nulo & No & Neutro/leve \\
Pioglitazona & ALT - GGT - AST & $\mathrm{Si}$ & $\mathrm{Si}$ & $\mathrm{Si}$ & Aumento \\
GLP1-RA & $\mathrm{ALT}-\mathrm{GGT}$ & $\mathrm{Si}$ & $\mathrm{Si}$ & $\mathrm{No}$ & Disminución \\
iSGLT2 & $\mathrm{ALT}-\mathrm{GGT}$ & $\mathrm{Si}$ & $\mathrm{n} / \mathrm{e}$ & $\mathrm{No}$ & Disminución \\
iDPP4 & $\mathrm{ALT}$ & $\mathrm{No}$ & $\mathrm{n} / \mathrm{e}$ & $\mathrm{No}$ & Neutro \\
\hline
\end{tabular}




\section{Recomendaciones}

13. La metformina debe considerarse una opción de primera linea para el tratamiento de la DM2 e HGNA. Aunque el beneficio para el tratamiento de la EHNA es marginal, el uso de metformina podría impactar sobre la mortalidad, tasas de descompensación y desarrollo de HCC en pacientes con HGNA y fibrosis avanzada.

14. Los fármacos agonistas del receptor de GLP-1, en especial liraglutida y semaglutida, exhiben efectos positivos sobre la histología de pacientes con HGNA y son promisorios para el manejo de esta enfermedad, especialmente en sujetos obesos.

15. En la actualidad, el empleo de agonistas del receptor de GLP-1 podría considerarse de elección como segunda línea, como adición a metformina, para el tratamiento de pacientes diabéticos con HGNA. El uso de agonistas del receptor de GLP-1 de administración semanal podría mejorar la adherencia en estos pacientes.

16. El uso de iSGLT2 en pacientes con EHNA, es promisorio pero la evidencia disponible no es robusta para esta indicación.

17. Con la actual evidencia, los iDPP4 no deberían ser recomendados para el manejo específico de EHNA, ya que la mayoría de los estudios no mostraron mejoría en la esteatosis hepática ni en la fibrosis.

\section{7. ¿Qué drogas con mecanismo de acción distinto a las antidiabéticas son estudiadas y han demostrado beneficio en HGNA?}

\section{Vitamina E}

Estudio PIVENS en no diabéticos, demostró que vitamina E $800 \mathrm{UI} /$ día fue superior a placebo (49\% vs $19 \%$ ) en mejorar EHNA, balonamiento, inflamación y esteatosis, sin empeoramiento en la fibrosis ${ }^{43}$. Estudio en pacientes con HGNA y DM 2, la vitamina E fue eficaz en reducir solo la esteatosis, pero no demostró reducir la actividad inflamatoria histológica a diferencia de su uso en terapia combinada con pioglitazona donde sí logró mejorar la histología ${ }^{44}$. Existen alertas respecto a su seguridad en uso a largo plazo con aumento de morbimortalidad, riesgo de accidente cerebrovascular hemorrágico y cáncer de próstata ${ }^{45}$.

\section{Ácido obeticólico (AO)}

Estudio FLINT mostró que mejora la histología de EHNA disminuyendo la fibrosis. Estudio REGENERATE (fase 3), mostró que AO (25 mg/ día) mejora significativamente fibrosis y actividad histológica en EHNA ${ }^{46}$. Otros agonistas tienen resultados prometedores ${ }^{47}$.

\section{Estatinas}

Pravastatina, atorvastatina y rosuvastatina han mostrado mejoría en las transaminasas, pero no en histología hepática ${ }^{2,45}$. Se ha reportado que son subprescritas en enfermedad hepática crónica debido al potencial riesgo de hepatotoxicidad que es muy bajo.

\section{Hormona tiroidea (Levotiroxina) y Análogos de hormona tiroidea (Resmetirom)}

En DM2 con HGNA, existe sólo un estudio de uso de levotiroxina en dosis bajas que logró disminuir contenido lipídico intrahepático ${ }^{2,45}$.

Resmetirom, es un agonista selectivo del receptor $B$ de hormona tiroidea con efectos benéficos en HGNA. Estudio fase $2 \mathrm{~b}$ evidenció disminuir contenido lipídico intrahepático, actualmente en estudio fase 3 (MAESTRO-NASH trial) ${ }^{48}$.

\section{Vitamina $D$}

Una revisión sistemática evidenció que los estudios de suplementación de vitamina $\mathrm{D}$ en HGNA son heterogéneos, de bajo poder estadístico y no concluyentes ${ }^{49}$.

\section{Probióticos (PB)}

Una revisión sistemática de tres estudios clínicos no demostró eficacia de probióticos en pacientes con HGNA. Faltan estudios que evalúen su efecto en marcadores histológicos de HGNA ${ }^{2,50,51}$.

\section{Recomendaciones}

18. Se necesitan más estudios controlados randomizados para recomendar el uso de vitamina E como terapia para la regresión de EHNA en pacientes diabéticos.

19. El ácido obeticólico es una droga potencialmente efectiva para el tratamiento de EHNA. Se requiere estudios a largo plazo para su uso en pacientes que tengan DM2 asociada. 
20. No existe evidencia actual para el uso de estatinas como tratamiento de HGNA, sin embargo, su uso en DM2 debe promoverse y prescribirse como parte del manejo integral para disminución del RCV cuando corresponda.

21. No hay suficiente evidencia actual para recomendar el uso de agonistas del receptor de hormona tiroidea, suplementación con vitamina D ni uso de probióticos en pacientes con HGNA y DM2.

8. ¿Cuál es el rol del médico de atención primaria e internista, del diabetólogo y del gastroenterólogo/hepatólogo en el manejo del HGNA en población diabética?

1. Médico general/familiar/internista: Rol fundamental en pesquisa de HGNA, manejo de FRCV, pesquisa FA y derivación.

- Pesquisa:

- Población: DM2.

- Método: Ecografía abdominal y aminotransferasas.

- Frecuencia: Pruebas de laboratorio hepático anual, ecografía abdominal cada 3 años ${ }^{52}$.
- Intervención:

- Pacientes con HGNA-aminotransferasas normales:

- Evaluar y aconsejar abstinencia de alcohol.

- Riesgo de FA (Algoritmo 2):

- Si riesgo bajo:Re-estratificar el riesgo en forma anual ${ }^{53}$

- Si riesgo intermedio o alto: Derivación a gastroenterología.

- Manejo de FRCV (Algoritmo 3).

- Derivación (Tablas 3 y 4).

2. Diabetólogo: Manejo integral de DM2 complejo.

- Pesquisa DM2 sin diagnóstico de HGNA.

- Riesgo FA (Algoritmo 2).

- Manejo FRCV (Algoritmo 3).

- Manejo de pacientes sin FA con objetivos metabólicos no logrados luego de intervención integral en medicina general/familiar/interna.

- Preferir fármacos con beneficio probado CV y favorables en manejo de HGNA.

- Derivación de pacientes con riesgo de fibrosis intermedio y alto a hepatología.

- Corroborar seguimiento por hepatología de pacientes que correspondan.

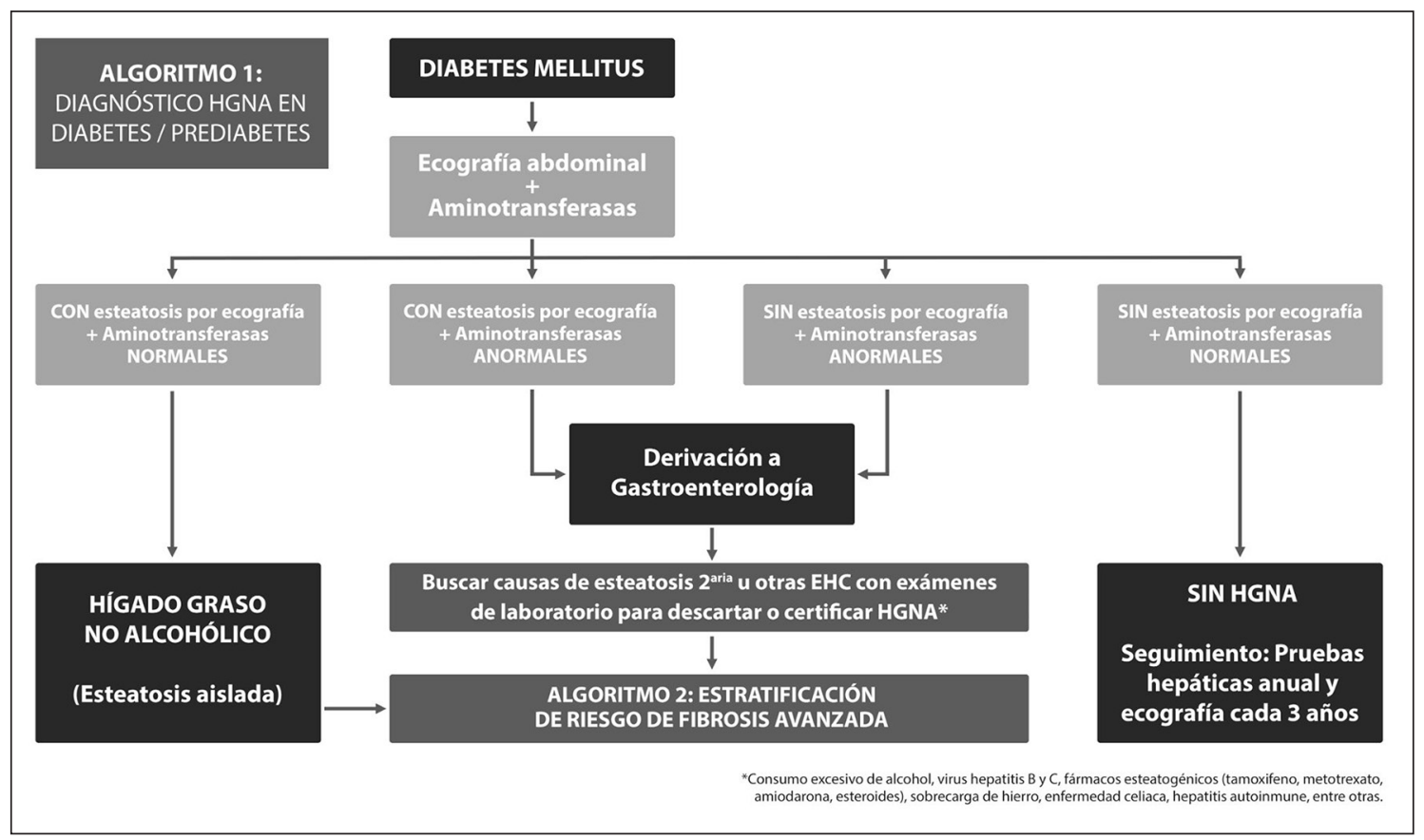

Figura 1. Algoritmo de evaluación de la esteatosis hepática en sujetos con diabetes mellitus o prediabetes. EHC: Enfermedad Hepática Crónica. 


\section{Tabla 3. Criterios de derivación a gastroenterólogo/hepatólogo}

Aminotransferasas alteradas en 2 o más controles con presencia o no de esteatosis en ecografía

Ecografía con signos de cirrosis

Riesgo intermedio o alto de fibrosis avanzada por métodos serológicos no invasivos

\section{Tabla 4. Criterios de derivación a Diabetólogo}

Falla en lograr metas de control metabólicas luego de intervención integral que incluyan fármacos con beneficio

Pacientes con criterio de derivación a gastroenterólogo
3. Gastroenterólogo/Hepatólogo: Evitar progresión de esteatohepatitis y fibrosis. Manejo de acuerdo al grado de daño.

- Evaluación de pacientes con riesgo intermedio o alto de FA por puntuación serológica: solicitar elastografía hepática.

- En FA realizar tratamiento con drogas hepatoespecíficas aprobadas y en protocolo si existiese.

- Pesquisa hipertensión portal y HCC en cirróticos conocidos.

- Manejo integral del cirrótico.

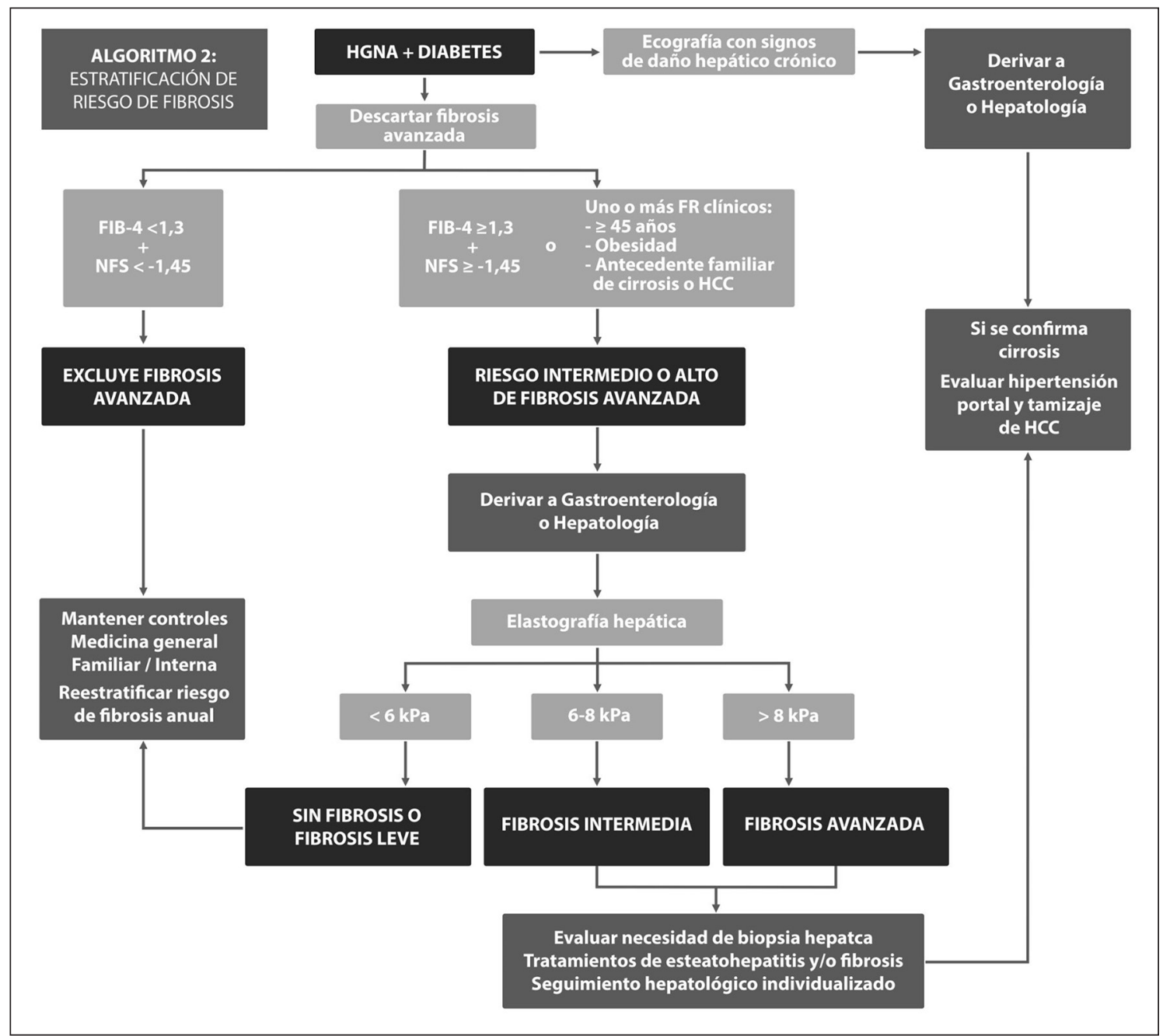

Figura 2. Algoritmo de estratificación de riesgo de fibrosis avanzada. HGNA: Enfermedad por hígado graso no alcohólico; FIB4: Índice de fibrosis hepática FIB4; NFS: NAFLD Fibrosis Score; FR: factor de riesgo; HCC: hepatocarcinoma, EH: Elastografía hepática. 


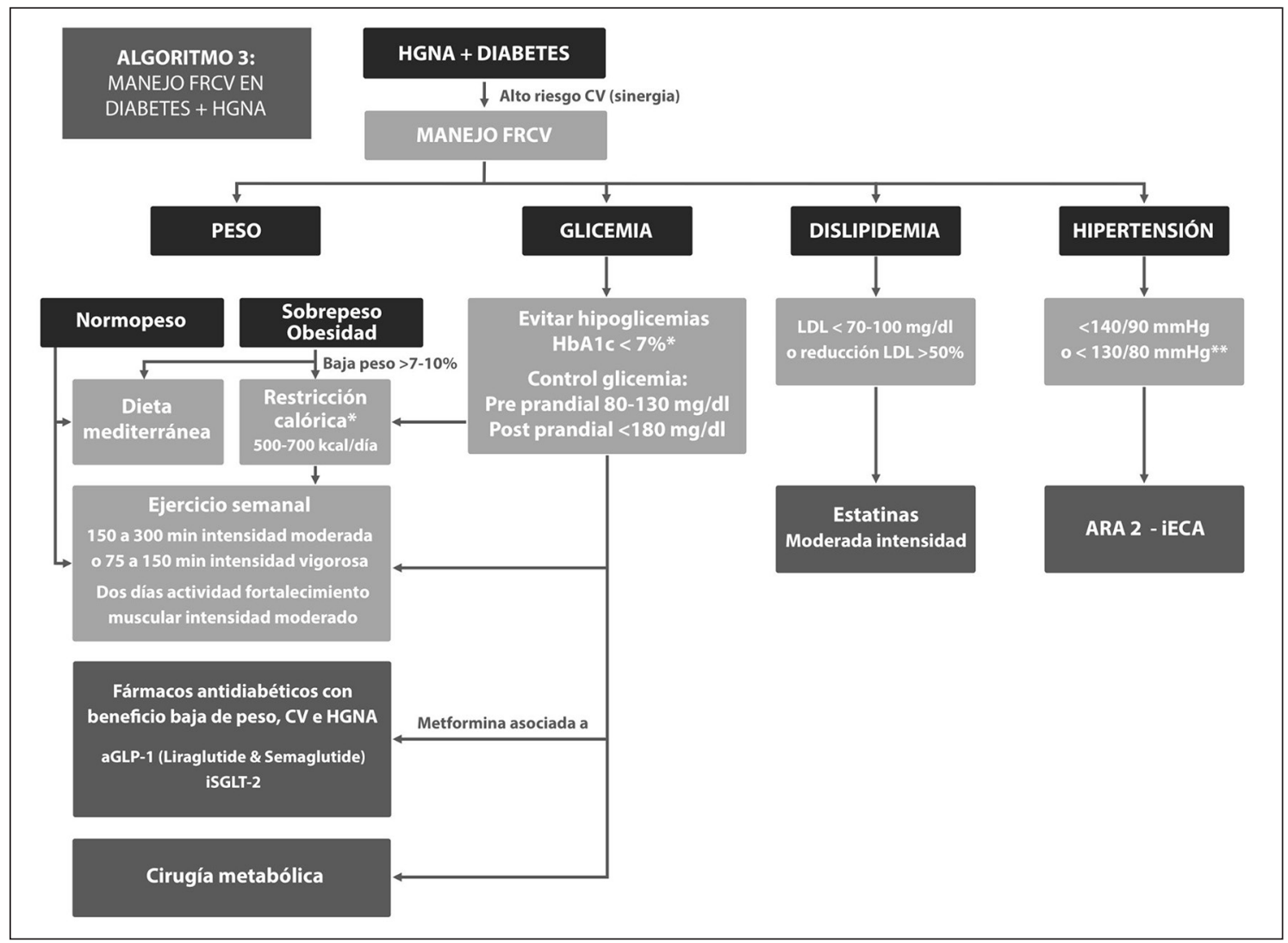

Figura 3. Algoritmo de manejo de FRCV en DM2 y HGNA. HGNA: enfermedad por hígado graso no alcohólico; CV: cardiovascular ; aGLP-1: análogo del péptido simil a glucagón tipo 1; iSGLT2: inhibidor del co-trasportador sodio-glucosa tipo 2; *HbA1c: hemoglobina glicosilada. Hipoglicemia: glicemia $<70 \mathrm{mg} / \mathrm{dl}$; ** optimizar metas 130/80 mmHg si enfermedad cardiovascular o riesgo > 15\% de enfermedad CV a 10 años (Framingham) ; ARA2: Antagonista de los receptores angiotensina; iECA: inhibidor enzima convertidora de angiotensina.

\section{Referencias}

1. Younossi ZM. Non-alcoholic fatty liver disease - A global public health perspective [Internet]. Journal of Hepatology. 2019; 70: 531-44. Available from: http:// dx.doi.org/10.1016/j.jhep.2018.10.033

2. Arab JP, Dirchwolf M, Álvares-da-Silva MR, Barrera F, Benítez C, Castellanos-Fernández $\mathrm{M}$, et al. Latin American Association for the study of the liver (ALEH) practice guidance for the diagnosis and treatment of non-alcoholic fatty liver disease. Ann. Hepatol. 2020; 19: 674-90.

3. Taylor RS, Taylor RJ, Bayliss S, Hagström H, Nasr P, Schattenberg JM, et al. Association Between Fibrosis Stage and Outcomes of Patients With Nonalcoholic Fatty Liver Disease: A Systematic Review and Meta-Analysis. Gastroenterology. 2020; 158: 1611-25.e12.
4. Méndez-Sánchez N, Arrese M, Gadano A, Oliveira CP, Fassio E, Arab JP, et al. The Latin American Association for the Study of the Liver (ALEH) position statement on the redefinition of fatty liver disease. Lancet Gastroenterol Hepatol. 2021; 6: 65-72.

5. Younossi ZM, Rinella ME, Sanyal AJ, Harrison SA, Brunt EM, Goodman Z, et al. From NAFLD to MAFLD: Implications of a Premature Change in Terminology [Internet]. Hepatology. 2021; 73: 1194-8. Available from: http://dx.doi.org/10.1002/hep.31420

6. Arrese M, Barrera F, Triantafilo N, Arab JP. Concurrent nonalcoholic fatty liver disease and type 2 diabetes: diagnostic and therapeutic considerations [Internet]. Expert Review of Gastroenterology \& Hepatology. 2019; 13: 849-66. Available from: http://dx.doi.org/10.1080/1 7474124.2019.1649981

7. American Diabetes Association. 10. Cardiovascular Di- 
sease and Risk Management: Standards of Medical Care in Diabetes-2019. Diabetes Care. 2019; 42: S103-23.

8. Smith BW, Adams LA. Nonalcoholic fatty liver disease and diabetes mellitus: pathogenesis and treatment. Nat. Rev. Endocrinol. 2011; 7: 456-65.

9. American Diabetes Association. 4. Comprehensive Medical Evaluation and Assessment of Comorbidities: Standards of Medical Care in Diabetes-2019. Diabetes Care. 2019; 42: S34-45.

10. Lomonaco R, Godinez Leiva E, Bril F, Shrestha S, Mansour L, Budd J, et al. Advanced Liver Fibrosis Is Common in Patients With Type 2 Diabetes Followed in the Outpatient Setting: The Need for Systematic Screening. Diabetes Care. 2021; 44: 399-406.

11. Perumpail BJ, Khan MA, Yoo ER, Cholankeril G, Kim D, Ahmed A. Clinical epidemiology and disease burden of nonalcoholic fatty liver disease. World J. Gastroenterol. 2017; 23: 8263-76.

12. Younossi ZM, Gramlich T, Matteoni CA, Boparai N, McCullough AJ. Nonalcoholic fatty liver disease in patients with type 2 diabetes. Clin. Gastroenterol. Hepatol. 2004; 2: 262-5.

13. Pais R, Charlotte F, Fedchuk L, Bedossa P, Lebray P, Poynard $\mathrm{T}$, et al. A systematic review of follow-up biopsies reveals disease progression in patients with non-alcoholic fatty liver. J. Hepatol. 2013; 59: 550-6.

14. Anstee QM, Darlay R, Cockell S, Meroni M, Govaere O, Tiniakos D, et al. Genome-wide association study of non-alcoholic fatty liver and steatohepatitis in a histologically characterised cohort. J. Hepatol. 2020; 73: 505-15.

15. Romeo S, Kozlitina J, Xing C, Pertsemlidis A, Cox D, Pennacchio LA, et al. Genetic variation in PNPLA3 confers susceptibility to nonalcoholic fatty liver disease. Nat. Genet. 2008; 40: 1461-5.

16. Méndez-Sánchez N, Cerda-Reyes E, Higuera de la Tijera F, Salas-García AK, Cabrera-Palma S, Cabrera-Álvarez $\mathrm{G}$, et al. Dyslipidemia as a risk factor for liver fibrosis progression in a multicentric population with non-alcoholic steatohepatitis. F1000Res. 2020; 9: 56.

17. Boursier J, Mueller O, Barret M, Machado M, Fizanne L, Araujo-Pérez F, et al. The severity of nonalcoholic fatty liver disease is associated with gut dysbiosis and shift in the metabolic function of the gut microbiota. Hepatology 2016; 63: 764-75.

18. Arab JP, Roblero JP, Altamirano J, Bessone F, Chaves Araujo R, Higuera-De la Tijera F, et al. Alcohol-related liver disease: Clinical practice guidelines by the Latin American Association for the Study of the Liver (ALEH). Ann. Hepatol. 2019; 18: 518-35.

19. Romero-Gómez M, Zelber-Sagi S, Trenell M. Treatment of NAFLD with diet, physical activity and exercise. J. Hepatol. 2017; 67: 829-46.

20. White DL, Kanwal F, El-Serag HB. Association between nonalcoholic fatty liver disease and risk for hepatocellular cancer, based on systematic review. Clin. Gastroenterol. Hepatol. 2012; 10: 1342-59.e2.

21. Younossi ZM, Stepanova M, Rafiq N, Makhlouf H, Younoszai Z, Agrawal R, et al. Pathologic criteria for nonalcoholic steatohepatitis: Interprotocol agreement and ability to predict liver-related mortality. Hepatology 2011; 53: 1874-82.

22. Arrese M, Barrera F, Triantafilo N, Arab JP. Concurrent nonalcoholic fatty liver disease and type 2 diabetes: diagnostic and therapeutic considerations. Expert Rev. Gastroenterol. Hepatol. 2019; 13: 849-66.

23. Castera L, Friedrich-Rust M, Loomba R. Noninvasive Assessment of Liver Disease in Patients With Nonalcoholic Fatty Liver Disease [Internet]. Gastroenterology. 2019; 156: 1264-81.e4. Available from: http://dx.doi. org/10.1053/j.gastro.2018.12.036

24. Arab JP, Barrera F, Arrese M. The Evolving Role of Liver Biopsy in Non-alcoholic Fatty Liver Disease. Ann. Hepatol. 2018; 17: 899-902.

25. Tilg H, Moschen AR, Roden M. NAFLD and diabetes mellitus [Internet]. Nature Reviews Gastroenterology \& Hepatology 2017; 14: 32-42. Available from: http:// dx.doi.org/10.1038/nrgastro.2016.147

26. Misciagna G, del Pilar Díaz M, Caramia DV, Bonfiglio C, Franco I, Noviello MR, et al. Effect of a low glycemic index Mediterranean diet on non-alcoholic fatty liver disease. A randomized controlled clinici trial [Internet]. The journal of nutrition, health \& aging. 2017; 21: 40412. Available from: http://dx.doi.org/10.1007/s12603016-0809-8

27. Ascha MS, Hanouneh IA, López R, Tamimi TA-R, Feldstein AF, Zein NN. The incidence and risk factors of hepatocellular carcinoma in patients with nonalcoholic steatohepatitis. Hepatology 2010; 51: 1972-8.

28. Bambha K, Wilson LA, Unalp A, Loomba R, Neuschwander-Tetri BA, Brunt EM, et al. Coffee consumption in NAFLD patients with lower insulin resistance is associated with lower risk of severe fibrosis. Liver Int. 2014; 34: 1250-8.

29. Hashida R, Kawaguchi T, Bekki M, Omoto M, Matsuse $\mathrm{H}, \mathrm{Nago} \mathrm{T}$, et al. Aerobic vs. resistance exercise in non-alcoholic fatty liver disease: A systematic review. J. Hepatol. 2017; 66: 142-52.

30. Lassailly G, Caiazzo R, Ntandja-Wandji L-C, Gnemmi V, Baud G, Verkindt H, et al. Bariatric Surgery Provides Long-term Resolution of Nonalcoholic Steatohepatitis and Regression of Fibrosis. Gastroenterology 2020; 159: 
1290-301.e5.

31. Said A, Akhter A. Meta-Analysis of Randomized Controlled Trials of Pharmacologic Agents in Non-alcoholic Steatohepatitis. Ann. Hepatol. 2017; 16: 538-47.

32. Vilar-Gómez E, Vuppalanchi R, Desai AP, Gawrieh S, Ghabril M, Saxena R, et al. Long-term metformin use may improve clinical outcomes in diabetic patients with non-alcoholic steatohepatitis and bridging fibrosis or compensated cirrhosis. Aliment. Pharmacol. Ther. 2019; 50: 317-28.

33. Vilar-Gómez E, Calzadilla-Bertot L, Wong VW-S, Castellanos M, Aller-de la Fuente R, Eslam M, et al. Type 2 Diabetes and Metformin Use Associate With Outcomes of Patients With Nonalcoholic Steatohepatitis-Related, Child-Pugh A Cirrhosis. Clin. Gastroenterol. Hepatol. 2021; 19: 136-45.e6.

34. Mahady SE, Webster AC, Walker S, Sanyal A, George J. The role of thiazolidinediones in non-alcoholic steatohepatitis - A systematic review and meta analysis [Internet]. Journal of Hepatology 2011; 55: 1383-90. Available from: http://dx.doi.org/10.1016/j.jhep.2011.03.016

35. Khan RS, Bril F, Cusi K, Newsome PN. Modulation of Insulin Resistance in Nonalcoholic Fatty Liver Disease [Internet]. Hepatology. 2019; Available from: http:// dx.doi.org/10.1002/hep.30429

36. Musso G, Cassader M, Paschetta E, Gambino R. Thiazolidinediones and Advanced Liver Fibrosis in Nonalcoholic Steatohepatitis: A Meta-analysis. JAMA Intern. Med. 2017; 177: 633-40.

37. Association AD, American Diabetes Association. 9. Pharmacologic Approaches to Glycemic Treatment: Standards of Medical Care in Diabetes-2021 [Internet]. Diabetes Care. 2021; 44: S111-24. Available from: http:// dx.doi.org/10.2337/dc21-s009

38. Mantovani A, Petracca G, Beatrice G, Csermely A, Lonardo A, Targher G. Glucagon-Like Peptide-1 Receptor Agonists for Treatment of Nonalcoholic Fatty Liver Disease and Nonalcoholic Steatohepatitis: An Updated Meta-Analysis of Randomized Controlled Trials [Internet]. Metabolites. 2021; 11: 73. Available from: http:// dx.doi.org/10.3390/metabo11020073

39. Armstrong MJ, Gaunt P, Aithal GP, Barton D, Hull D, Parker R, et al. Liraglutide safety and efficacy in patients with non-alcoholic steatohepatitis (LEAN): a multicentre, double-blind, randomised, placebo-controlled phase 2 study [Internet]. The Lancet. 2016; 387: 67990. Available from: http://dx.doi.org/10.1016/s01406736(15)00803-x

40. Harrison SA, Calanna S, Cusi K, Linder M, Okanoue T, Ratziu V, et al. Semaglutide for the treatment of non-alcoholic steatohepatitis: Trial design and comparison of non-invasive biomarkers [Internet]. Contemporary Clinical Trials. 2020; 97: 106174. Available from: http:// dx.doi.org/10.1016/j.cct.2020.106174

41. Coelho F dos S, dos Santos Coelho F, Borges Canha M, von Hafe M, Neves JS, Vale C, et al. Effects of sodium glucose co transporter 2 inhibitors on liver parameters and steatosis: A meta analysis of randomized clinical trials [Internet]. Diabetes/Metabolism Research and Reviews. 2020; Available from: http://dx.doi.org/10.1002/ dmrr.3413

42. Kim K-S, Lee B-W. Beneficial effect of anti-diabetic drugs for nonalcoholic fatty liver disease. Clin. Mol. Hepatol. 2020; 26: 430-43.

43. Sanyal AJ, Chalasani N, Kowdley KV, McCullough A, Diehl AM, Bass NM, et al. Pioglitazone, vitamin E, or placebo for nonalcoholic steatohepatitis. N. Engl. J. Med. 2010; 362: 1675-85.

44. Bril F, Biernacki DM, Kalavalapalli S, Lomonaco R, Subbarayan SK, Lai J, et al. Role of Vitamin E for Nonalcoholic Steatohepatitis in Patients With Type 2 Diabetes: A Randomized Controlled Trial. Diabetes Care. 2019; 42: 1481-8.

45. Marjot T, Moolla A, Cobbold JF, Hodson L, Tomlinson JW. Nonalcoholic Fatty Liver Disease in Adults: Current Concepts in Etiology, Outcomes, and Management. Endocr. Rev. [Internet]. 2020; 41. Available from: http:// dx.doi.org/10.1210/endrev/bnz009

46. Younossi ZM, Ratziu V, Loomba R, Rinella M, Anstee QM, Goodman Z, et al. Obeticholic acid for the treatment of non-alcoholic steatohepatitis: interim analysis from a multicentre, randomised, placebo-controlled phase 3 trial. Lancet. 2019; 394: 2184-96.

47. Lucas KJ, López P, Lawitz E, Sheikh A, Aizenberg D, Hsia S, et al. Tropifexor, a highly potent FXR agonist, produces robust and dose-dependent reductions in hepatic fat and serum alanine aminotransferase in patients with fibrotic NASH after 12 weeks of therapy: FLIGHT-FXR Part C interim results [Internet]. Digestive and Liver Disease. 2020; 52: e38. Available from: http://dx.doi.org/10.1016/j.dld.2019.12.129

48. Harrison SA, Bashir MR, Guy CD, Zhou R, Moylan CA, Frias JP, et al. Resmetirom (MGL-3196) for the treatment of non-alcoholic steatohepatitis: a multicentre, randomised, double-blind, placebo-controlled, phase 2 trial. Lancet. 2019; 394: 2012-24.

49. Barchetta I, Cimini FA, Cavallo MG. Vitamin D and Metabolic Dysfunction-Associated Fatty Liver Disease (MAFLD): An Update. Nutrients [Internet]. 2020; 12. Available from: http://dx.doi.org/10.3390/nu12113302

50. Loguercio C, Federico A, Tuccillo C, Terracciano F, D’Auria MV, De Simone C, et al. Beneficial effects of a 
probiotic VSL\#3 on parameters of liver dysfunction in chronic liver diseases. J. Clin. Gastroenterol. 2005; 39: 540-3.

51. Jayakumar S, Loomba R. Review article: emerging role of the gut microbiome in the progression of nonalcoholic fatty liver disease and potential therapeutic implications. Aliment. Pharmacol. Ther. 2019; 50: 144-58.

52. European Association for the Study of the Liver (EASL),
European Association for the Study of Diabetes (EASD), European Association for the Study of Obesity (EASO). EASL-EASD-EASO Clinical Practice Guidelines for the Management of Non-Alcoholic Fatty Liver Disease. Obes. Facts. 2016; 9: 65-90.

53. Petroni ML, Brodosi L, Bugianesi E, Marchesini G. Management of non-alcoholic fatty liver disease. BMJ. 2021; 372:m4747. 